\title{
Throughput and reliability analysis of a scalable broadcast MAC for distributed wireless networks
}

\author{
Subodh Pudasaini ${ }^{1}$, Seokjoo Shin ${ }^{1 *}$ and Kiseon Kim ${ }^{2}$
}

\begin{abstract}
In carrier-sense-multiple-access-based distributed wireless networks, the legacy mechanism of arbitrating the access attempts of multiple broadcast users (using a fixed-size temporal contention window consisting of multiple contention slots having uniform selection probability) has been well understood to be inefficient in avoiding collisions, especially when the network is large. Recently, it has been found that this inefficiency can be mitigated by exploiting an additional degree of freedom (DoF) over the contention window, referred to as contention slot selection distribution (CSSD). In this article, the authors examine how the additional DoF contributes to enhancing network performance and quantify the performance gains that the network can achieve in terms of easily understandable performance indicators like network throughput efficiency and broadcast reliability. A discrete-time Markov chain is formulated to characterize the access arbitration process involving a reverse exponential CSSD and its steady-state solution is fed to a renewal-reward process-based network model to estimate the performances of interest. Validated numerical results are presented which confirm that this new channel access arbitration mechanism can significantly enhance the scalability of distributed broadcast networks.
\end{abstract}

Keywords: Broadcast MAC, Scalability, Reliability, Contention slot selection distribution, Distributed wireless network, IEEE 802.11

\section{Introduction}

Medium access control (MAC)-layer broadcasting is a fundamental network-wide communication primitive that provides an operational platform for many other higherlayer protocols in distributed wireless networks [1]. A very common example is the route discovery mechanism in general-purpose multi-hop ad hoc networks [2]. With the advent of different special purpose ad hoc networks like sensor networks [3] and vehicular ad hoc networks [4], the scope of broadcasting has been widened to incorporate mechanism to disseminate sensed or monitored physical phenomena of interest.

The basic distributed coordination function (DCF), defined in Section 9.2.7 of the IEEE 802.11 protocol specification [5], has been used as a de facto MAC for broadcast applications in distributed wireless networks.

*Correspondence: sjshin@chosun.ac.kr

1 Department of Computer Engineering, Chosun University, 375-Seosuk-dong, Dong-Gu, Gwangju 501-759, Korea

Full list of author information is available at the end of the article
Hereafter, it will be referred to as a B-MAC. It uses a temporal contention window-based channel arbitration mechanism along with carrier sense multiple access (CSMA). Note that the rationale behind using the contention window, even after performing carrier sensing, is to mandate contending users to access the channel only after a further deferral (random backoff) period which would possibly avoid concurrent transmissions (literally, packet collisions) from multiple contending users. Note that the efficiency of such a collision avoidance mechanism depends on two factors: the size of the contention window and the number of contending users. For a given size of the contention window, the collision probability increases with an increase in the contending population size. As a consequence, the throughput efficiency of the network decreases. Moreover, because of the large number of destinations in broadcast operation, unlike the single destination in unicast operation, simultaneously acknowledging reception from multiple destinations is not feasible. Therefore, the transmitting user cannot

\section{包 Springer}

(C) 2012 Pudasaini et al.; licensee Springer. This is an Open Access article distributed under the terms of the Creative Commons Attribution License (http://creativecommons.org/licenses/by/2.0), which permits unrestricted use, distribution, and reproduction in any medium, provided the original work is properly cited. 
know whether the transmitted packet has safely reached its destinations. Thus, B-MAC does not invoke MAC layer retransmission or recovery for the collided packets as its unicast counterpart does. Therefore, broadcast reliability (in terms of successful reception at destinations) also quickly decreases with an increase in contending population size.

Based on these considerations (the inverse relationship of network throughput efficiency and broadcast reliability with contending population size), it can be stated that BMAC is nonscalable. The ideal prerequisite for any MAC to be scalable would be its ability to maintain the performances of interest intact, irrespective of any increase in contending population size. Hence, from the perspective of MAC layer, it is desirable to reduce collisions as much as possible to attain scalability. An interesting concept has been proposed in [6,7] which can drastically reduce the number of collisions in the network. The concept simply exploits an additional degree of freedom (DoF) in designing a broadcast MAC. In particular, the uniform contention slot selection distribution (CSSD) over the contention window is replaced with a nonuniform CSSD which multiplicatively increases the probability of picking a later slot relative to an earlier slot. As a result, the degree of contention remains low for the earlier slots within the contention window, irrespective of the contending population size, and therefore the number of collisions can greatly be reduced. Hereafter, a B-MAC with such a nonuniform CSSD will be called a scalable B-MAC (SB-MAC).

The number of applications of IEEE 802.11-based broadcast networks has continuously been increasing. The accurate characterization of underlying MAC protocols in such networks is essential not only to analyze their performance, but also to identify ways to upgrade and optimize them. In response to this need, $\mathrm{Ma}$ and Chen [8] have provided a simple analytical model for BMAC, which has been further enhanced by Wang and Hassan [9]. Even though these models accurately estimate the performance of B-MAC, they cannot directly be used to characterize the performance of SB-MAC because SBMAC not only incorporates the nonuniform CSSD, but also adds the concept of resetting the backoff counter when it finds a channel busy instead of freezing it as in the conventional B-MAC. Therefore, in this article, a new analytical model is proposed which accommodates both these modifications and has a simple closed-form solution.

The rest of the article is organized as follows: in the following section, the conventional B-MAC is reviewed, and its inability to support network scalability is comprehensively analyzed. In Section "SB-MAC", the SB-MAC and an analytical model to characterize its performance are presented. Numerical and simulation results are presented in Section "Performance results and discussions". Finally, in the last section, some concluding remarks are provided.

\section{Legacy broadcast MAC (B-MAC) Operational procedure of B-MAC}

Figure 1 depicts the legacy B-MAC as defined in Section 9.2.7 of the IEEE 802.11 protocol specification [5]. According to this MAC, each user with broadcast packet(s) to transmit first monitors the channel activity. If the channel is found to be idle for a period of time equal to the distributed interframe space (DIFS), the MAC selects a random backoff time from a set of uniformly distributed prospective backoff durations that are bounded in the interval $[0, W-1]$, where $W$ is the size of the contention window, for which the user should defer transmission. It then decreases the backoff timer by one at the passage of every idle slot until either the channel becomes busy again or the backoff timer reaches zero. If the timer has not yet reached zero but the channel has been detected as busy, the user freezes its timer. The countdown starts again after once more detecting the channel as idle for the DIFS interval. When the timer has finally decremented to zero, the user transmits the packet.

Note that in B-MAC, the recipients do not acknowledge reception as in a unicast MAC. Therefore, there is no MAC layer recovery or retransmission. For this reason, IEEE 802.11 has specified a fixed contention window for broadcast traffic which is equal to the initial (minimum) contention window specified for unicast traffic.

\section{Discouraging throughput-reliability tradeoff}

Recall that the efficiency of the access arbitration method used in B-MAC depends on two factors: the number of contending users and the size of contention window. For a given (specified) contention window size, collision probability increases with an increase in the contending population size. Using a large contention window could be a

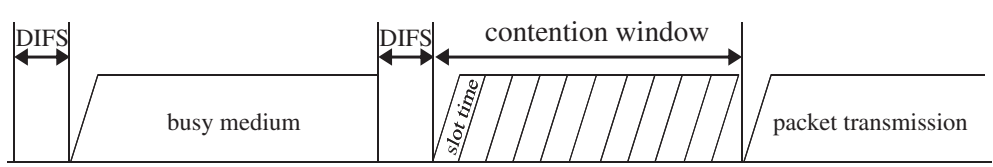

Figure 1 Basic channel access mechanism in the legacy B-MAC. 
trivial approach to reduce collisions. This approach, however, has counterproductive implications in the network performance.

Figure 2 shows the performance ${ }^{\mathrm{a}}$ of IEEE 802.11a distributed networks for varying contention window sizes. It is evident that, for a given contending population size, both throughput efficiency and broadcast reliability increase with an increase in contention window size up to a certain threshold, let the size be called the critical contention window. Beyond this critical value of contention window, throughput is traded off against reliability. In other words, throughput starts to decrease even though broadcast reliability manages to increase.

Note that even though the usage of a large contention window (larger than the critical window) maintains graceful throughput efficiency, especially when the number of users is high, it is still not a good strategy to use such a large window because it severely elongates transmission delays, which is strongly undesirable for delay-sensitive broadcast traffics.

\section{SB-MAC}

An ideal prerequisite for any MAC to be scalable would be its ability to maintain the performances of interest (e.g., network throughput efficiency and broadcast reliability) intact, irrespective of any increase in contending population size. Note that both these performance indicators are inversely proportional to the number of collisions, while the number of collisions itself is directly proportional to the contending population size. Hence, from the perspective of MAC layer, collisions should be lowered as much as possible to attain scalability.

Note that the most readily available design choice to enhance the collision avoidance efficiency of B-MAC is to

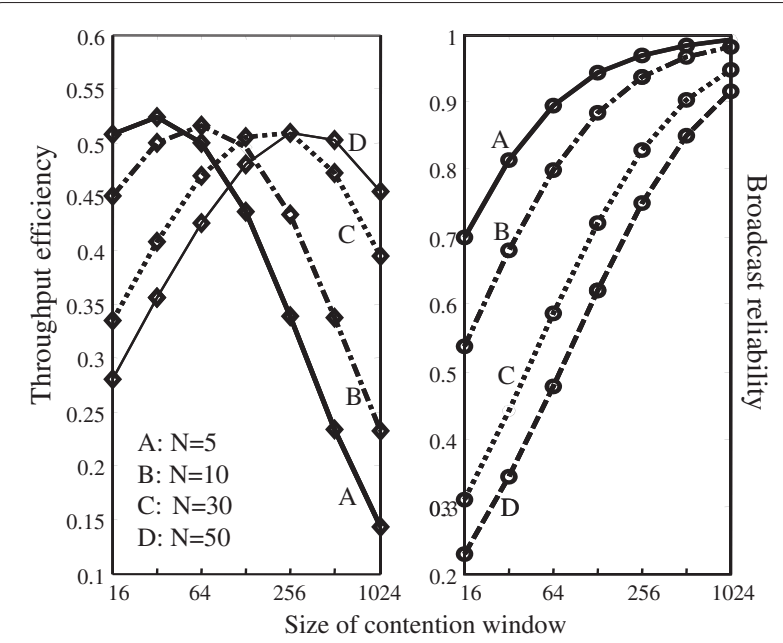

Figure 2 Throughput-reliability tradeoff of B-MAC with variable contention window sizes. increase the size of contention window. This implies that the number of DoF available for adjusting the contention window is one. SB-MAC adds a second DoF (referred to as CSSD over the contention window) to reduce the number of collisions in the network, irrespective of the contending population size.

\section{Operational procedure of SB-MAC}

Recall the operational procedure of the legacy B-MAC which was previously described in Section "Legacy broadcast MAC (B-MAC)". The operational procedure of the SB-MAC is exactly same as that of B-MAC, except for the following two changes:

- First, the way of choosing the random further-deferral (backoff) duration upon performing carrier sense is slightly different because the legacy uniform CSSD over the contention window has been replaced with the reverse-exponential CSSD [7] in (1)

$$
q_{k}=\frac{1-\alpha}{1-\alpha^{W}} a^{W-(k+1)} ; \quad k \in[0, W-1]
$$

where $k$ is any contention slot within the window and $\alpha$ is a design variable (an additional DoF) which is bounded over the open interval $(0,1)$. Note that $\lim _{\alpha \rightarrow 1} \frac{1-\alpha}{1-\alpha^{W}} a^{W-(k+1)}=\frac{1}{W}$. In other words the distribution in (1) mimics the legacy uniform CSSD when $\alpha \rightarrow 1$.

- Second, the requirement to freeze the backoff counter upon sensing the channel to be busy is relaxed. In other words, if the channel is detected to be busy during an ongoing backoff cycle, the backoff counter is reset, and the next backoff cycle is initiated.

\section{Performance analysis of SB-MAC}

In this section, an analytical model is presented to estimate the performances of interest: network throughput efficiency and broadcast reliability. The model consists of two sub-models: a newly developed Markov chainbased user model that describes the details of SB-MAC channel arbitration, and a widely used renewal-reward process-based network model [10] that estimates the performances of interest based on the solution obtained from the Markovian user model.

\section{Markov chain-based user model}

Figure 3 shows the discrete time Markov chain (DTMC) model which characterizes the backoff procedure of the SB-MAC for a given contention window size $W$. The DTMC has been developed based on the assumption that the packet arrival rate at the transmission buffer is high enough that the buffer will never be empty. 


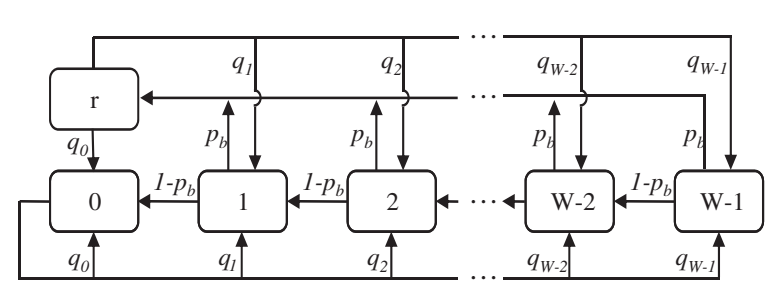

Figure 3 Representation of the channel access arbitration of the SB-MAC using a DTMC.

In the DTMC, there are all together $W+1$ number of states; state 0 to $W-1$ correspond to the backoff counter values of the tagged user, while an auxiliary state $r$ corresponds to the situation where the backoff counter has been reset. Using conventional state transition notations, all possible transitions in the DTMC and their corresponding transition probabilities can be written as follows:

(a) $P\{k \mid k+1\}=1-p_{b} ; k \in[0, W-2]$,

(b) $P\{r \mid k\}=p_{b} ; k \in[1, W-1]$,

(c) $P\{k \mid 0\}=q_{k} ; k \in[0, W-1]$,

(d) $P\{k \mid r\}=P\{k \mid 0\} ; k \in[0, W-1]$.

The first expression corresponds to the event that the backoff timer is decreased by one upon detecting the channel to be idle, while the second expression corresponds to the event that the backoff counter is reset upon finding the channel to be busy. In these expressions, $p_{b}$ is the probability that the channel is found to be busy. Its mathematical definition is given in (8). The last two expressions correspond to the events that a random slot is selected for initiating the backoff procedure upon completing the previous backoff cycle (backoff counter reached zero) and upon resetting the backoff counter (channel is detected to be busy), respectively. The transition probabilities are equal in those last two expressions.

In the DTMC, let the stationary distribution of the probabilities that a tagged user is in state $k, k \in[0, W-1]$, and $r$ be $b_{k}$ and $b_{r}$, respectively. It will be shown that a closed-form solution exists for this DTMC. Note that

$$
b_{k}= \begin{cases}\frac{1-\alpha}{1-\alpha^{W}} \alpha^{W-(k+1)}\left\{b_{0}+b_{r}\right\}+\left(1-p_{b}\right) b_{k+1} ; & 0 \leq k \leq W-2, \\ \frac{1-\alpha}{1-\alpha^{W}}\left\{b_{0}+b_{r}\right\} ; & k=W-1\end{cases}
$$

and

$$
\begin{aligned}
b_{r} & =p_{b}\left(b_{1}+b_{2}+\cdots+b_{W-1}\right) \\
& =p_{b}\left(1-b_{0}-b_{r}\right) \\
& =\frac{p_{b}\left(1-b_{0}\right)}{1+p_{b}} .
\end{aligned}
$$

To express (2) with a single expression for all $k$, it can be rewritten in a different style. For the special case when $W$ is $4, b_{k}$ for $k \in[0,3]$ can be rewritten, after few steps of manipulations, as follows:

$$
\begin{aligned}
b_{3} & =\left(\frac{\alpha}{1-p_{b}}\right)^{0}\left(1-p_{b}\right)^{0} z, \\
b_{2} & =\left[\left(\frac{\alpha}{1-p_{b}}\right)^{0}+\left(\frac{\alpha}{1-p_{b}}\right)^{1}\right]\left(1-p_{b}\right)^{1} z, \\
b_{1} & =\left[\left(\frac{\alpha}{1-p_{b}}\right)^{0}+\left(\frac{\alpha}{1-p_{b}}\right)^{1}+\left(\frac{\alpha}{1-p_{b}}\right)^{2}\right]\left(1-p_{b}\right)^{2} z, \\
b_{0} & =\left[\left(\frac{\alpha}{1-p_{b}}\right)^{0}+\left(\frac{\alpha}{1-p_{b}}\right)^{1}+\left(\frac{\alpha}{1-p_{b}}\right)^{2}+\left(\frac{\alpha}{1-p_{b}}\right)^{3}\right] \\
& \cdot\left(1-p_{b}\right)^{3} z
\end{aligned}
$$

where $z=\frac{1-\alpha}{1-\alpha^{4}}\left(b_{r}+b_{0}\right)$. Therefore, jointly considering the relations in (3) and (4), a recursive relation of $b_{k}$ in (2) can simply be expressed as follows:

$$
b_{k}=\frac{b_{0}+p_{b}}{1+p_{b}} \frac{1-\alpha}{1-\alpha^{W}}\left(1-p_{b}\right)^{W-(k+1)} \sum_{j=k}^{W-1}\left(\frac{\alpha}{1-p_{b}}\right)^{W-(j+1)} \text {. }
$$

From (3) and (4), it is evident that the probability of being in any of the states in the DTMC can be represented as a function of $b_{0}$. Therefore, $b_{0}$ can be obtained using the following normalization condition:

$$
\sum_{k=0}^{W-1} b_{k}+b_{r}=1
$$

Upon solving (5)

$$
b_{0}=\frac{\left(\Upsilon-p_{b}\right)-(\Upsilon-1)\left(1+p_{b}\right)}{\left(\Upsilon-p_{b}\right)},
$$

where

$$
\begin{aligned}
\Upsilon= & \frac{1-\alpha}{1-\alpha^{W}} \cdot \frac{1}{\alpha-\left(1+p_{b}\right)} \\
& \cdot\left[\frac{\alpha^{W+1}-1}{\alpha-1}+\frac{\left(1-p_{b}\right)^{W+1}-1}{p_{b}}\right] .
\end{aligned}
$$

The probability of transmission $(\tau)$ of the tagged user in a generic slot is equal to $b_{0}$ because it is allowed to transmit only when its backoff counter reaches zero. Then, given the parameter $\tau$, the probability $p_{b}$ that the channel remains busy when there are $N$ contending users in the network is given by

$$
p_{b}=1-(1-\tau)^{N} .
$$


Rearranging (6) and (8), the following nonlinear system can be defined and solved numerically to get the values of the two unknowns $\tau$ and $p_{b}$ :

$$
\left\{\begin{array}{l}
\tau-b_{0}=0 \\
p_{b}-1+(1-\tau)^{N}=0
\end{array}\right.
$$

\section{Renewal-reward process-based network model}

Given the transmission probability $\tau$, performances of interest, including network throughput efficiency and broadcast reliability, can be calculated using the renewal reward process-based network model in which the packet transmission process is approximated by a renewal process [10]. In such an approximation, transmission attempts from $N$ contending users, each of which transmits packet with probability $\tau$, are considered to be independent events which repeat over time. The outcome space for every attempt is $\{I, S, C\}$, where $I$ corresponds to the event that there is no access attempt (the channel is idle), $S$ corresponds to the event that the attempt was successful, and $C$ corresponds to the event that the attempt ended in a collision. Considering the outcome space for the events, the inter event duration $T$ can be estimated as follows:

$$
E[T]=\sum_{\forall x \in(I, S, C)} P_{x} T_{x}
$$

where $P_{x}$ is the probability that the outcome of the event is $x$ and $T_{x}$ is the duration for which $x$ lasts. With the assumption that all $N$ contending users can hear each others transmission (i.e., connected single-hop network), $P_{x}$ can be obtained as follows:

$$
P_{x}= \begin{cases}(1-\tau)^{N} ; & x=I \\ N \tau(1-\tau)^{N-1} ; & x=S \\ 1-P_{I}-P_{S} ; & x=C\end{cases}
$$

Moreover, $T_{x}$ can be obtained as in [8] considering the signaling mechanism specified for broadcast DCF in [5]:

$$
T_{x}= \begin{cases}\sigma ; & x=I \\ \left(L_{H}+E[P L]\right) / R_{d}+D I F S+\delta ; & x=S \text { or } C\end{cases}
$$

where $\sigma$ is the duration of a physical slot, $E[P L]$ is the expected payload length, $L_{H}$ is the time required to transmit header (both PHY and MAC header), $R_{d}$ is the raw data rate of the channel, and $\delta$ is the propagation delay.

For a given $N$, nonlinear system in (9) can be solved numerically to get two unknowns $\tau$ and $p_{b}$. Based on these values, $P_{x}$ in (11) can be calculated. For a given $E[P], T_{x}$ in (12) can be obtained. Once both $P_{x}$ and $T_{x}$ have been obtained, $E[T]$ in (10) can be calculated. Upon estimation of $E[T]$, network throughput efficiency ( $\Im$ ) can be calculated using the expected reward $E[R]$ per renewal event duration $E[T]$ as follows:

$$
\mathfrak{s}=\frac{E[R]}{E[T]},
$$

where $E[R]=P_{s} \cdot E[P L]$. Likewise, the broadcast reliability $(\Re)$, the probability that the transmitted packet does not collide with other packets, can be calculated easily using the following relation when the probability of success $\left(P_{s}\right)$ in the renewal packet transmission process is known:

$$
\Re=\frac{P_{s}}{N \tau} \text {. }
$$

\section{Performance results and discussions \\ Model validation}

A custom discrete-event simulator is developed in MAT$\mathrm{LAB}$ which can adequately represent the channel access arbitration details of the legacy B-MAC. By making some necessary changes, it is used to simulate SB-MAC. Note that the analytical model derived in the previous section is independent of any specific PHY-layer technology. Hence, detailed PHY implementations have intentionally been ignored in the simulator as developed here.

For the purpose of model validation, the numerically obtained results have been compared with the simulation results. For both numerical analysis and simulation, a channel error-free distributed network with no hiddenterminals has been used consisting of a varying number of broadcast users, each having a never-empty MAC queue (this situation literally corresponds to the case of saturated packet arrival). Note that collisions are the only reason of packet loss in the aforementioned settings. Unless otherwise stated, the following are the default parameters (with corresponding values in parentheses) used in the current study: channel rate $(6 \mathrm{Mbps})$; standard contention window size (16); physical slot duration ( $9 \mu \mathrm{s})$; PHY header (20 $\mu \mathrm{s})$; MAC header (28 Bytes); DIFS (34 $\mu \mathrm{s})$; payload PL (128 Bytes); and propagation delay $(1 \mu \mathrm{s})$.

As can be noted from Table 1, the simulated results ${ }^{b}$ show good match with the analytical results for all configurations of contention window size, contending population size, $\alpha$, and payload length. This confirms that the analytical model as developed here is accurate enough to be used for quick and easy numerical performance analysis.

\section{Comparison of SB-MAC and B-MAC}

Once the accuracy of the analytical model had been validated, it was used to generalize the results ( $\mathfrak{s}$ and $\mathfrak{R}$ ) for different network parameters. The generalized results were then compared with the corresponding performance results of B-MAC considering two different cases: (1) 
Table 1 Comparison of analytical and simulation results

\begin{tabular}{|c|c|c|c|c|c|c|c|}
\hline \multirow[t]{2}{*}{$\mathbf{N}$} & \multirow[t]{2}{*}{ w } & \multirow[t]{2}{*}{ PL } & \multirow[t]{2}{*}{$\alpha$} & \multicolumn{2}{|c|}{$\mathfrak{\Im}$} & \multicolumn{2}{|c|}{$\Re$} \\
\hline & & & & Ana. & Sim. & Ana. & Sim. \\
\hline \multirow[t]{3}{*}{5} & 16 & 128 & 0.4 & 0.4939 & 0.487 & 0.9012 & 0.907 \\
\hline & & & 0.6 & 0.4989 & 0.491 & 0.8947 & 0.903 \\
\hline & & & 0.8 & 0.5121 & 0.509 & 0.8705 & 0.881 \\
\hline \multirow[t]{3}{*}{20} & 16 & 128 & 0.4 & 0.5107 & 0.502 & 0.8241 & 0.828 \\
\hline & & & 0.6 & 0.5122 & 0.504 & 0.8104 & 0.815 \\
\hline & & & 0.8 & 0.5098 & 0.497 & 0.7446 & 0.749 \\
\hline \multirow[t]{3}{*}{40} & 32 & 256 & 0.4 & 0.6379 & 0.629 & 0.8899 & 0.893 \\
\hline & & & 0.6 & 0.6397 & 0.631 & 0.8864 & 0.891 \\
\hline & & & 0.8 & 0.6465 & 0.640 & 0.8691 & 0.871 \\
\hline \multirow[t]{3}{*}{60} & 32 & 256 & 0.4 & 0.6425 & 0.639 & 0.8785 & 0.882 \\
\hline & & & 0.6 & 0.6440 & 0.639 & 0.8746 & 0.879 \\
\hline & & & 0.8 & 0.6493 & 0.641 & 0.8536 & 0.858 \\
\hline
\end{tabular}

standard contention window, and (2) nonstandard contention window.

\section{Case 1: standard contention window}

Recall that $\alpha$ is the protocol parameter in SB-MAC. It can be assigned any value greater than zero and less than one. The selection of $\alpha$ value, however, has immediate implications for the performances of interest, $\mathfrak{\Im}$ and $\mathfrak{R}$, as shown in Figures 4 and 5.

From Figure 4, it is apparent that when there are few number of users in the network, roughly on the

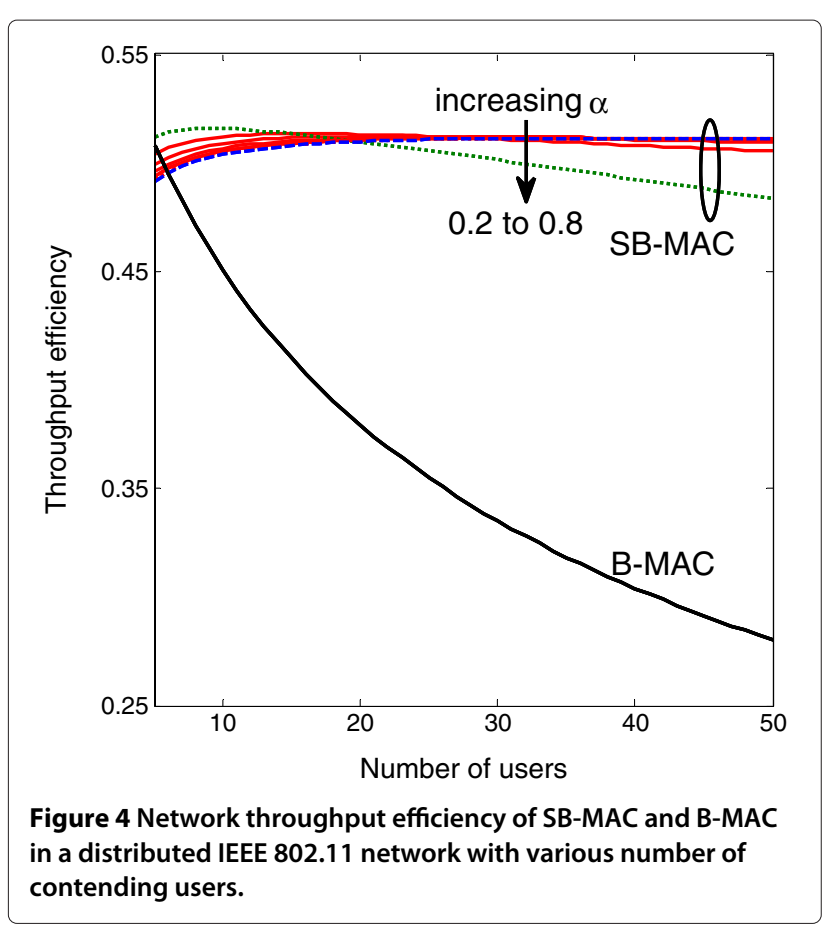

order of contention window size, it is beneficial to use higher value of $\alpha$ because it can maintain higher $\mathfrak{s}$. For example, among the considered $\alpha$ values, 0.2 to 0.8 , $\Im$ attains its maximum when $\alpha$ is 0.8 and the number of contending users is less than or roughly equal to the size of contention window (16). As the ratio of contending users over contention size increases, it is beneficial to use lower values for $\alpha$ to maintain a graceful $\Im$.

As shown in Figure 5, it would be desirable to use lower $\alpha$ value to enhance $\Re$ in SB-MAC. The lower the value of

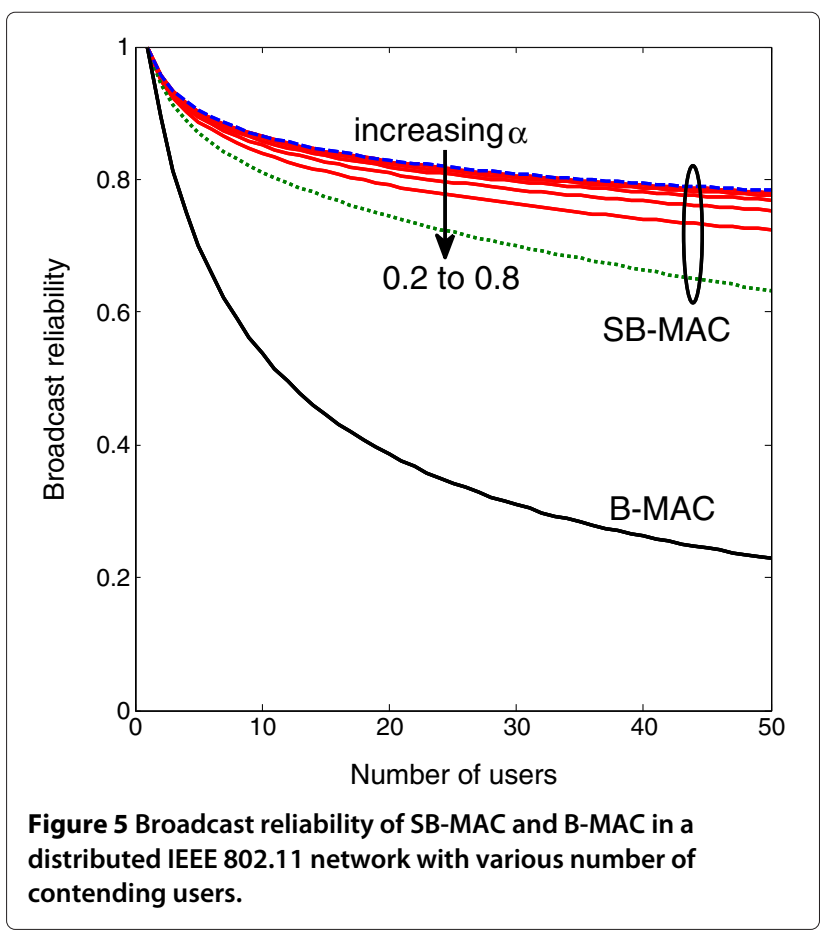


$\alpha$, the higher will be the chance of selecting collision-free earlier contention slot. For example, among the various $\alpha$ values considered, reliability is best when $\alpha$ is 0.2 . The desirability of lower $\alpha$ values for better $\mathfrak{R}$ is common to $\Im$ as well, expect for the case when the contending population size is less than (or in the order of) the size of the contention window used.

Beside the SB-MAC performance in terms of $\mathfrak{s}$ and $\Re$, Figures 4 and 5 also show these performance for the B-MAC. Contrary to the performance trend in SBMAC, both $\mathfrak{s}$ and $\mathfrak{R}$ in B-MAC severely decrease with an increase in contending population size. A simple comparison reveals that the SB-MAC performs better over the entire range of considered contending population, irrespective of the values specified for $\alpha$. It is interesting to highlight that the performance gain that SB-MAC offers over B-MAC increases with increase in contending population size. For example, SB-MAC offers an enhancement of approximately $230 \%$ in $\mathfrak{R}$ along with $75 \%$ enhancement in $\mathfrak{I}$ when the number of contending users is three times the size of the contention window.

\section{Case 2: nonstandard contention window}

$\mathfrak{I}$ and $\mathfrak{R}$ of B-MAC are compared with that of SB-MAC considering various non standard contention window sizes, up to either four times smaller or larger than that of the standard contention window (16), and a typical $\alpha$ value (0.2). Recall that, in Figure 2, $\Im$ of B-MAC linearly increases with respect to contention window size

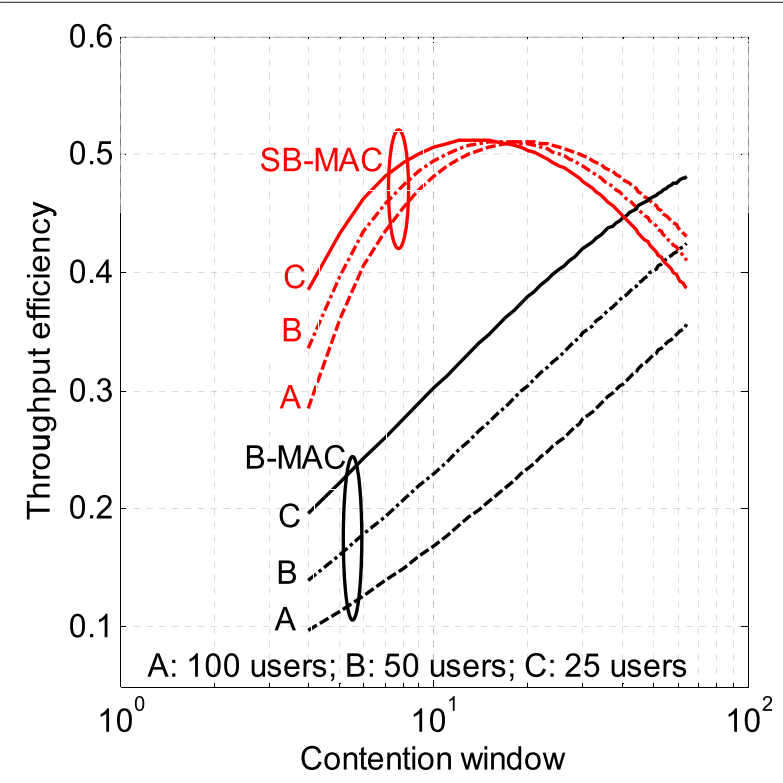

Figure 6 Network throughput efficiency of SB-MAC and B-MAC in a distributed IEEE 802.11 network with various contention window sizes. (for a given number of user) until it reaches the critical threshold. Beyond that threshold it starts to decrease. Similar critical threshold exists for SB-MAC as well, as can be noted from Figure 6, but it is relatively smaller than that of B-MAC. For example, when there are 50 users in the network, the critical window for B-MAC is almost 16 times larger than the standard contention window. In case of SB-MAC, it is nearly of the same order as the standard contention window. From Figure 6 it is evident that the performance of SB-MAC in terms of $\Im$ is superior than that of B-MAC for any number of user population size, especially for the contention windows that are equal or smaller than the critical contention window size. Unlike $\mathfrak{I}$, $\Re$ of SB-MAC monotonically increases, regardless of the number of users, with increase in the contention window sizes as can be noted from Figure 7. It is noteworthy to mention that $\Re$ of SB-MAC is higher than that of B-MAC for any cases of contention window and user population sizes.

\section{Conclusion}

Unlike the throughput-centric design objectives in unicast contention-based MAC protocols, their broadcast counterparts should have reliability-centric design objective because they do not necessarily have MAC layer retransmission mechanisms for the lost packets. From the perspective of a MAC layer, broadcast reliability issues can be addressed by reducing the number of packet collisions as much as possible. Recently, it has been noted that the number of collisions in broadcast CSMA/CA

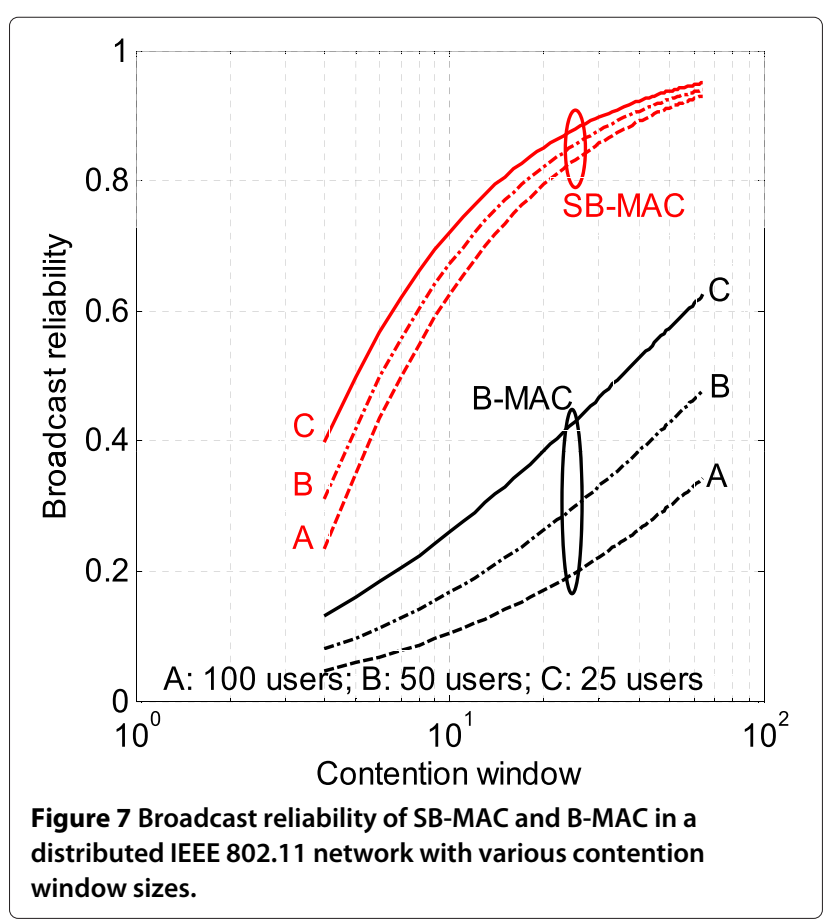


networks can significantly be reduced by using a well scaled reverse-exponential CSSD to conduct the backoff procedure. In this article, a simple and accurate analytical model has been developed to characterize the channel access arbitration according to this approach. The model was validated using computer simulations, and it was then used to demonstrate the performance gains available from this approach in terms of easily understandable performance indicators such as network throughput efficiency and broadcast reliability.

\section{Endnotes}

a The performance values of interest are obtained analytically using the model in [9]. The precise definitions of the performance matrices shown are available in Section "SB-MAC", and the assumed system parameters are summarized in Section "Performance results and discussions". $\mathrm{b}$ The simulation results presented here are the mean of the results obtained from one hundred independently repeated simulations.

\begin{abstract}
Abbreviations
B-MAC: broadcast MAC; CSMA: carrier sense multiple access; CSSD: contention slot selection distribution; DCF: distributed coordination function; DIFS: distributed inter frame space; DoF: degree of freedom; DTMC: discrete time Markov chain; MAC: medium access control; SB-MAC: scalable broadcast MAC.
\end{abstract}

\section{Competing interests}

The authors declare that they have no competing interests.

\section{Acknowledgements}

This research was supported in part by the Basic Science Research Program through the National Research Foundation of Korea (NRF)funded by the Ministry of Education, Science and Technology (2012006327), and it was also supported in part by the Basic Research Project through a grant provided by GIST, Grant K20903001804-11E0100-00910 funded by the Ministry of Education, Science, and Technology (MEST) and the World-Class University Program through the National Research Foundation of Korea (R31-10026).

\section{Author details}

${ }^{1}$ Department of Computer Engineering, Chosun University, 375-Seosuk-dong, Dong-Gu, Gwangju 501-759, Korea. ${ }^{2}$ Department of Nanobio Materials and Electronics, School of Information and Communication, Gwangju Institute of Science and Technology (GIST), Gwangju, Korea.

Received: 8 June 2011 Accepted: 16 July 2012

Published: 14 August 2012

\section{References}

1. B Wiliams, T Camp, Comparision of broadcasting technique for mobile Ad Hoc networks. in ACM Int Conf MobiHoc (New York, USA, 2002), pp. 194-205

2. H Zhang, Z Jiang, Modelling and performance analysis of ad hoc broadcasting schemes. Perform. Eval. 63(12), 1196-1215 (2006)

3. IF Akyildiz, W Su, Y Sankarasubramaniam, E Cayirci, Wireless sensor networks: a survey. Comput. Commun. 38(4), 393-422 (2002)

4. ML Sichitiu, M Khil, Inter-vehicle communication systems: a survey. IEEE Commun. Surv. Tutor. 10(2), 88-105 (2008)

5. IEEE Std. 802.11-2007, Part 11: wireless IAN medium access control and physical layer specifications, 2007

6. K Jamieson, H Balakrishnan, YC Tay, Sift: a MAC protocol for event-driven wireless sensor networks. Lecture Note Comput. Sci. 3868, 260-275 (2006)

7. YC Tay, K Jamieson, H Balakkrishnan, Collision-minimizing CSMA and its applications to wireless sensor networks. IEEE J. Sel. Areas Commun. 22(6), 1048-1057 (2004)
8. X Ma, X Chen, Performance analysis of IEEE 802.11 broadcast scheme in Ad Hoc wireless LANs. IEEE Trans. Veh. Technol. 57(6), 3757-3768 (2008)

9. Z Wang, M Hassan, Throughput-reliability tradeoff in 802.11-based vehicular saftey communications. in IEEE Int Conf CCNC (Las Vegas, USA, 2009), pp. 1-5

10. G Bianchi, Performance analysis of the IEEE 802.11 distributed coordination function. IEEE J. Sel. Areas Commun. 8(3), 535-547 (2000)

doi:10.1186/1687-1499-2012-254

Cite this article as: Pudasaini et al.: Throughput and reliability analysis of a scalable broadcast MAC for distributed wireless networks. EURASIP Journal on Wireless Communications and Networking 2012 2012:254.

\section{Submit your manuscript to a SpringerOpen ${ }^{\circ}$ journal and benefit from:}

- Convenient online submission

- Rigorous peer review

- Immediate publication on acceptance

- Open access: articles freely available online

- High visibility within the field

- Retaining the copyright to your article

Submit your next manuscript at $>$ springeropen.com 